\title{
An X-ray bright ERO hosting a type 2 QSO ${ }^{\star}$
}

\author{
P. Severgnini ${ }^{1}$, A. Caccianiga ${ }^{1}$, V. Braito ${ }^{1,2}$, R. Della Ceca ${ }^{1}$, T. Maccacaro ${ }^{1}$, M. Akiyama ${ }^{3}$, F. J. Carrera ${ }^{4}$, \\ M. T. Ceballos ${ }^{4}$, M. J. Page ${ }^{5}$, P. Saracco ${ }^{1}$, and M. G. Watson ${ }^{6}$
}

1 INAF - Osservatorio Astronomico di Brera (OAB), via Brera 28, 20121 Milano, Italy

e-mail: [paola; caccia; braito;rdc; tommaso; saracco]@brera.mi.astro.it

2 Exploration of the Universe Division, NASA Goddard Space Flight Center, Greenbelt Road, Greenbelt, MD 20771, USA e-mail: vale@milkyway.gsfc.nasa.gov

${ }^{3}$ Subaru Telescope, National Astronomical Observatory of Japan, 650 North A'ohoku Place, Hilo, HI 96720, USA e-mail: akiyama@subaru.naoj.org

4 Instituto de Fisica de Cantabria (CSIC-UC), Avenida de los Castros, 39005 Santander, Spain e-mail: [carreraf;ceballos]@ifca.unican.es

5 Mullard Space Science Laboratory, University College London, Holmbury St. Mary, Dorking, Surrey RH5 6NT, UK e-mail: mjp@mssl.ucl.ac.uk

6 X-ray Astronomy Group, Department of Physics and Astronomy, Leicester University, Leicester LE1 7RH, UK e-mail: mgw@star.le.ac.uk

Received 29 July 2005 / Accepted 4 February 2006

\section{ABSTRACT}

\begin{abstract}
We present the XMM-Newton and the optical-VLT spectra along with the optical and the near-infrared photometric data of one of the brightest X-ray $\left(F_{2-10 \mathrm{keV}} \sim 10^{-13} \mathrm{erg} \mathrm{s}^{-1} \mathrm{~cm}^{-2}\right)$ extremely red objects $(R-K \geq 5)$ discovered so far. The source, XBS J0216-0435, belongs to the XMM-Newton Bright Serendipitous Survey and it has extreme X-ray-to-optical ( 220) and X-ray-to-near-infrared ( 60) flux ratios. Thanks to its brightness, the X-ray statistics are good enough for an accurate spectral analysis by which the presence of an X-ray obscured $\left(N_{\mathrm{H}}>10^{22} \mathrm{~cm}^{-2}\right)$ QSO $\left(L_{2-10 \mathrm{keV}}=4 \times 10^{45} \mathrm{erg} \mathrm{s}^{-1}\right)$ is determined. A statistically significant $(\sim 99 \%)$ excess around $2 \mathrm{keV}$ in the observed-frame suggests the presence of an emission line. By assuming that this feature corresponds to the iron $\mathrm{K} \alpha$ line at $6.4 \mathrm{keV}$, a first estimate of the redshift of the source is derived $\left(z_{\mathrm{x}} \sim 2\right)$. The presence of a high redshift QSO2 has been finally confirmed through dedicated VLT optical spectroscopic observations $\left(z_{\mathrm{O}}=1.985 \pm 0.002\right)$. This result yields to an optical validation of a new X-ray Line Emitting Object (XLEO) for which the redshift has been firstly derived from the X-ray data. XBS J0216-0435 can be considered one of the few examples of X-ray obscured QSO2 at high redshift for which a detailed X-ray and optical spectral analysis has been possible. The spectral energy distribution from radio to X-rays is also presented. Finally from the near-infrared data the luminosity and the stellar mass of the host galaxy has been estimated finding a new example of the coexistence at high- $z$ between massive galaxies and powerful QSOs.
\end{abstract}

Key words. galaxies: active - X-rays: galaxies - galaxies: individual: XBS J0216-0435

\section{Introduction}

Extremely red objects $(R-K \geq 5$, EROs) with bright $\mathrm{X}$-ray fluxes $\left(F_{(2-10 \mathrm{keV})} \geq 10^{-14} \mathrm{erg} \mathrm{s}^{-1} \mathrm{~cm}^{-2}\right)$ and with high $\mathrm{X}$-ray-to-optical and X-ray-to-NIR (near-infrared) flux ratios $\left(F_{(2-10 \mathrm{keV})} / F_{\mathrm{opt}}, F_{(2-10 \mathrm{keV})} / F_{K} \gg 10\right)$ are among the best candidates to be obscured ${ }^{1}$ AGNs, i.e. the main ingredient of the X-ray Cosmic Background (Gilli et al. 2001a; Ueda et al. 2003). Indeed, given the minimum redshift observed for extragalactic EROs with such high flux ratios $(z \gtrsim 0.6$, see e.g. Akiyama et al. 2001; Mignoli et al. 2004; Severgnini et al. 2005a and references therein; Maiolino et al. 2006) an X-ray flux $\sim 10^{-14} \mathrm{erg} \mathrm{s}^{-1} \mathrm{~cm}^{-2}$ corresponds to $L_{2-10 \mathrm{keV}}>10^{43} \mathrm{erg} \mathrm{s}^{-1}$, typical of AGN. Moreover, a factor of at least 10 higher in the $F_{(2-10 \mathrm{keV})} / F_{\text {opt }}$ and $F_{(2-10 \mathrm{keV})} / F_{K}$ ratios than those observed

$\star$ Based on data obtained with the European Southern Observatory (VLT program 075.B-0229(A); NTT program 074.A-0024(A)) and with the Telescopio Nazionale Galileo.

${ }^{1}$ We use here $N_{\mathrm{H}}=10^{22} \mathrm{~cm}^{-2}$ and $E(B-V) \geq 1.7 \mathrm{mag}$ as dividing values between unabsorbed and absorbed sources in the $\mathrm{X}$-ray and optical bands respectively. for unobscured AGNs $\left(F_{(2-10 \mathrm{keV})} / F_{\mathrm{opt}}, F_{(2-10 \mathrm{keV})} / F_{K} \sim 1\right)$ can be easily justified by invoking the presence of an obscuring optically-thick medium (e.g. molecular torus) along the line of sight. In this case, while the UV and optical emission is totally or heavily suppressed by the large amount of dust, the X-ray emission is less affected by the absorbing medium producing high $F_{(2-10 \mathrm{keV})} / F_{\text {opt }}$ and $F_{(2-10 \mathrm{keV})} / F_{K}$. If the above scenario is true, it implies that the selection of EROs with such high flux ratios at bright X-ray fluxes $\left(F_{(2-10 \mathrm{keV})} \geq 10^{-13} \mathrm{erg} \mathrm{s}^{-1} \mathrm{~cm}^{-2}\right)$ is an extremely efficient way to find the long sought high luminosity $\left(L_{(2-10 \mathrm{keV})}>10^{44} \mathrm{erg} \mathrm{s}^{-1}\right)$, X-ray obscured type $2 \mathrm{QSOs}^{2}$ (hereafter QSO2).

In this paper we present XMM-Newton and VLT optical spectroscopic data of a very red X-ray selected source (XBS J0216-0435) belonging to the XMM-Newton Bright Serendipitous Survey (XMM-BSS, Della Ceca et al. 2005; Della Ceca et al. 2004; Caccianiga et al. 2004; Severgnini et al. 2003). This source, given its bright X-ray flux

2 The "type 2" denomination refers to the optical spectral properties of the AGNs characterized only by strong and highly ionized narrow $\left(F W H M<1000 \mathrm{~km} \mathrm{~s}^{-1}\right)$ emission lines. 
and its high $F_{(2-10 \mathrm{keV})} / F_{\text {opt }}$ and $F_{(2-10 \mathrm{keV})} / F_{K}$, represents the prototype of the EROs in which a QSO2 is expected. The good $\mathrm{X}$-ray statistics have made the spectral analysis possible and allowed us to give a first estimate of the redshift of the source $(z \sim 2$, Sect. 3). The X-ray properties found (the spectral shape, the amount of absorption and the presence of the FeK line) are typical of an obscured QSO. The presence of a high redshift QSO2 has been finally confirmed through dedicated VLT optical spectroscopic observations (Sect. 4).

Throughout this paper we assume $H_{0}=65 \mathrm{~km} \mathrm{~s}^{-1} \mathrm{Mpc}^{-1}$ and $\Omega_{M}=03, \Omega_{\Lambda}=07$. All the magnitudes are in the Vega system.

\section{An ERO with extreme $F_{(2-10 \mathrm{keV})} / F_{\mathrm{opt}}$ and $\boldsymbol{F}_{(2-10 \mathrm{keV})} / \boldsymbol{F}_{\mathrm{K}}$ flux ratios}

XBS J0216-0435 belongs to the XMM-BSS sample. The sample definition and selection criteria are described in Della Ceca et al. (2004). The X-ray source discussed here is in the XMM-Newton Subaru field SXDS4 ${ }^{3}$ and has a count rate in the $0.5-4.5 \mathrm{keV}$ band of $(1.32 \pm 0.12) \times 10^{-2}$ counts/s which corresponds to a $F_{(0.5-4.5 \mathrm{keV})} \sim 10^{-13} \mathrm{erg} \mathrm{cm}^{-2} \mathrm{~s}^{-1}$ (see Della Ceca et al. 2004). Contrary to the majority ( $\sim 90 \%$ ) of the XMM-BSS sources, the optical counterpart of XBS J0216-0435 is much fainter than the POSS II limit ( $R \sim 21 \mathrm{mag})$.

Our own $R$-band and $K$-band photometric observations have been performed on January 2005 at the ESO New Technology Telescope (NTT) using the ESO Multi-Mode Instrument (EMMI, $1 \mathrm{~h}$ of exposure time) and on October 2002 at the Telescopio Nazionale Galileo (TNG) using the Near Infrared Camera Spectrometer (NICS, 15 min of exposure) respectively. Both the observing runs were carried out under similar seeing conditions $\left(\sim 1.2^{\prime \prime}\right)$. The optical and NIR images were reduced using standard iraf routines. The zero-points have been derived by measuring the instrumental magnitudes of standard stars observed just before and/or after the scientific target and assuming the average extinction reported in the Observatory web pages. Astrometric calibration was performed using the software package GAIA (version 2.6-9 by P.W. Draper) by matching sources found in the USNO catalogue (Monet et al. 1998). The typical uncertainty in the best fit solution is about $1^{\prime \prime}$. A weak $(R \sim 24.5$ mag, see Fig. 1, left panel) optical counterpart is visible within 4 arcsec from the X-ray position of the source (corresponding to the $90 \%$ confidence level X-ray error circle, see Della Ceca et al. 2004). Within the same distance from the X-ray position, two possible weak NIR objects are visible with a $K^{\prime} \sim 19.5 \mathrm{mag}$ (see Fig. 1, right panel). The two NIR sources are separated by $\sim 1^{\prime \prime}$. Even though the quality of the data is not good enough to allow a morphological analysis, all the sources appear extended. We have positionally registered the $R$ to the $K^{\prime}$ image by using the 8 brightest sources in the field. We find that the optical source is spatially coincident with the southern NIR source. This latter is also the NIR source closest to the X-ray centroid position (less than $1^{\prime \prime}$ ). Given the color of the two sources $\left(R-K^{\prime} \sim 5\right.$ for the southern source and $R-K^{\prime}>6$ for the northern one) these objects can be classified as EROs. Although the different optical-to-NIR colors of the two objects suggest that they could have different redshifts, the presence of a group of galaxies can not be ruled out. However, even in the case of a group, the X-ray emission observed is dominated by a powerful AGN (see Sect. 3) which, on the basis of

\footnotetext{
3 No Subaru data are available since the object is outside the Suprime-cam deep survey field.
}
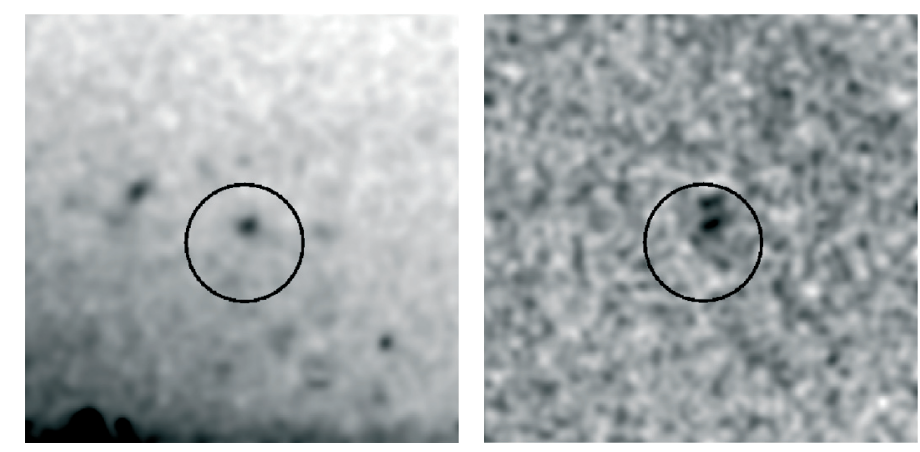

Fig. 1. $R$-band (left panel) and $K$-band (right panel) smoothed images $\left(30^{\prime \prime} \times 30^{\prime \prime}\right)$ of XBS J0216-0435. North is to the top and east to the left. The circle of $4^{\prime \prime}$ of radius marks the centroid of the X-ray position. The strong gradient present in the optical image is due to a very bright star ( $R=9 \mathrm{mag}$ ) near the objects considered here.

VLT optical spectroscopic observations (see Sect. 4), is hosted in the southern ERO. This source is the object with the highest $F_{(2-10 \mathrm{keV})} / F_{\text {opt }}{ }^{4}(\sim 220)$ among the XMM-BSS sources (see Fig. 6 in Della Ceca et al. 2004). Furthermore, taking into account the 2-10 keV X-ray emitting EROs collected from the literature by Brusa et al. (2005), XBS J0216-0435 is one of the EROs with the highest X-ray flux among those with the highest $F_{(2-10 \mathrm{keV})} / F_{\text {opt }}$ (filled circle in Fig. 2) and X-ray-to-NIR ratios (XBS J0216-0435 has a $F_{(2-10 \mathrm{keV})} / F_{K}{ }^{5} \simeq 60$, see for comparison Fig. 4 of Brusa et al. 2005). We note that, as described in the next section, we have derived the X-ray flux from two different $X M M$-Newton observations separated by more than two years. The extremely high $F_{(2-10 \mathrm{keV})} / F_{\text {opt }}\left(F_{K}\right)$ ratios quoted above are not likely due to an intrinsic variation of the source since the $\mathrm{X}$ ray fluxes measured in the two observations are consistent within the errors.

\section{XMM-Newton data}

\subsection{X-ray observations}

XBS J0216-0435 was serendipitously observed by the EPIC cameras on 8 August 2000 (OBS ID $=0112371701, \sim 24 \mathrm{ks}$ exposure) and on 7 January 2003 (OBS ID $=0112372001, \sim 28 \mathrm{ks}$ exposure). The observations were carried out in full frame mode and with the thin filter applied. The data have been processed using the SAS (Science Analysis Software) version 5.4. The latest calibration files and response matrices released by the EPIC team have been used to create new response matrices that include also the correction for the effective area at the source position in the detector (off-axis $\sim 8$ arcmin). Events files released from the standard pipeline have been filtered from high background time intervals and only events corresponding to patterns $0-12$ and $0-4$ have been used for the MOS and pn respectively. In the first of the two exposures, XBS J0216-0435 lies on a gap of the pn camera and only the MOS data are available. The net exposure times after data cleaning are reported in Table 1.

At the spatial resolution of XMM-Newton EPIC instruments, our source is consistent with a point-like object. This result does

\footnotetext{
${ }^{4} F_{(2-10 \mathrm{keV})}$ has been derived from the $F_{(0.5-4.5 \mathrm{keV})}$ by assuming an observed $\Gamma=1.5$ on the basis of the X-ray colors, see Della Ceca et al. (2004). $F_{\text {opt }}$ is the integrated flux in the Cousin $R$ band system. More specifically: $F_{\text {opt }}=1568 \AA \times\left(2.15 \times 10^{-9} \operatorname{cgs} \AA^{-1} \times 10^{\left(-0.4 \cdot m_{R}\right)}\right)$, see Fukugita et al. (1995).

5 For the $K$-band we adopted the magnitude to flux conversion used in Brusa et al. (2005).
} 


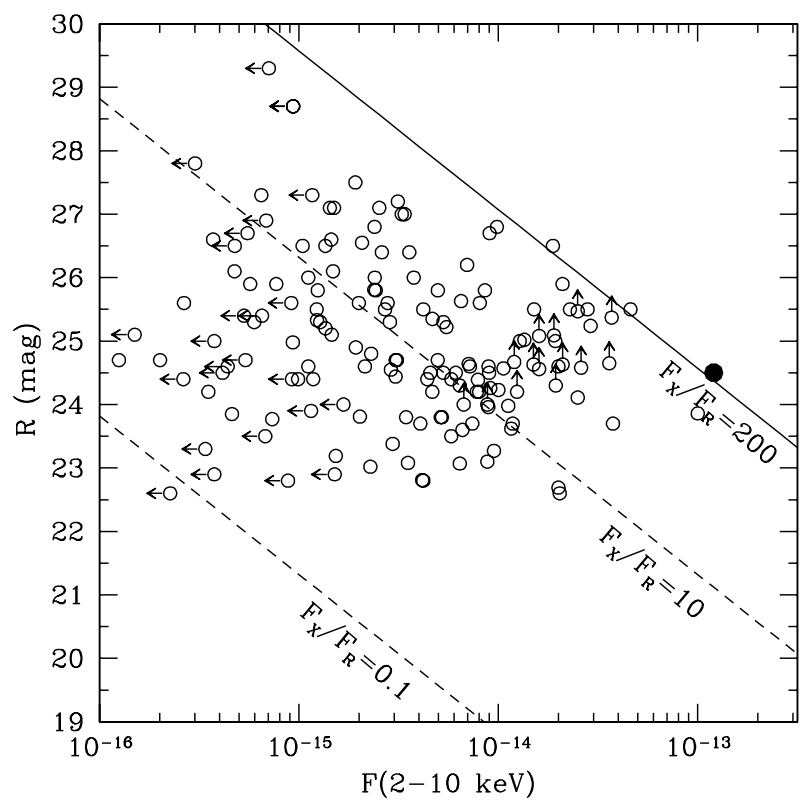

Fig. 2. $R$ magnitude vs. 2-10 keV flux (in units of erg $\mathrm{cm}^{-2} \mathrm{~s}^{-1}$ ) for XBS J0216-0435 (filled circle) and for other X-ray emitting EROs (empty circles) taken from the literature (Mignoli et al. 2004; Mainieri et al. 2002; Stevens et al. 2003; Alexander et al. 2002, 2003; Barger et al.2003; Roche et al. 2003; Szokoly et al. 2004; Willott et al. 2003; Gandhi et al. 2004; Severgnini et al. 2005a; Brusa et al. 2005). Upper and lower limits of the $(2-10 \mathrm{keV})$ and $R$ magnitudes are marked with arrows. The two dashed lines define the region where unobscured type 1 AGNs typically lie (see e.g. Maccacaro et al. 1988; Fiore et al. 2003).

Table 1. XMM-Newton observations of XBS J0216-0435.

\begin{tabular}{ccc}
\hline \hline $\begin{array}{c}\text { Obs. ID } \\
011237\end{array}$ & EPIC-cameras & $\begin{array}{c}\text { Net exposure time } \\
{[\mathrm{ks}]}\end{array}$ \\
\hline 1701 & MOS1+MOS2 & $\sim 39$ \\
\hline 2001 & pn & $\sim 22$ \\
& MOS1+MOS2 & $\sim 52$ \\
\hline
\end{tabular}

not discard the presence of a group of galaxies or a small cluster (core radius of order of $90 \mathrm{kpc}$, Mohr et al. 1999).

\section{2. $X$-ray spectral analysis}

The spectral analysis has been performed using XSPEC 11.2.0. The X-ray spectrum was extracted using a circular region of $28^{\prime \prime}$ of radius in all the images except for the MOS data of the second observation where a smaller aperture (21" of radius) has been used because of the proximity of a CCD gap. In all cases the background spectra were extracted from $\sim 4$ times larger sourcefree circular regions close to the object. In order to improve the statistics, MOS1 and MOS2 data of each observation have been combined together and, finally, MOS and pn spectra have been binned to have at least 20 total counts per energy channel. The MOS and pn spectra of the second observation were fitted simultaneously in the $0.5-10 \mathrm{keV}$ band leaving free the relative normalizations. Total net counts of $\sim 250$ and $\sim 740$ have been accumulated in the first and second observation respectively. In the fitting procedure, the appropriate Galactic hydrogen column density along the line of sight $\left(2.37 \times 10^{20} \mathrm{~cm}^{-2}\right)$ has been taken into account (Dickey \& Lockman 1990). In the rest of the paper,

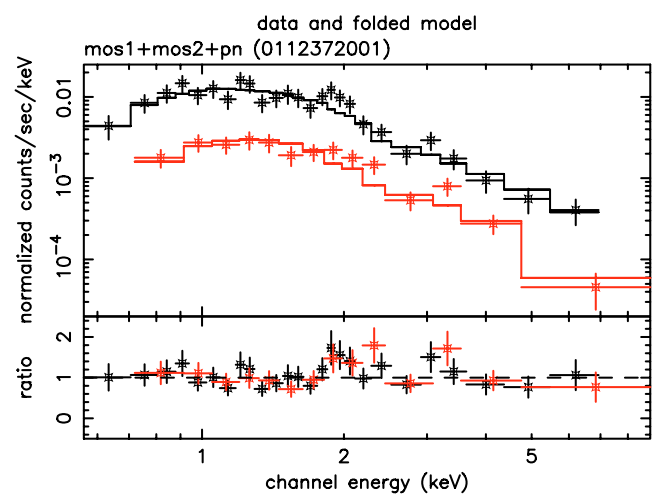

Fig. 3. Mos+pn folded X-ray spectrum of XBS J0216-0435 (upper panel, solid points) obtained during the second (0112372001) observation. The data are fitted by a single absorbed power-law model (continuous lines). Ratio between data and model is plotted in the lower panel as a function of energy.

and unless stated otherwise, errors are given at the $90 \%$ confidence level for one interesting parameter $\left(\Delta \chi^{2}=2.71\right)$.

The photometric optical, NIR and X-ray properties of XBS J0216-0435 suggest that the observed X-ray emission could be due to the presence of an obscured AGN and/or a group or a small cluster of galaxies. While a pure thermal component (mekal thermal plasma model, Mewe et al. 1985; Kaastra 1992; Liedahl et al. 1995) is rejected by the fit (assuming solar abundances and $k T<10 \mathrm{keV}$ the resulting $\chi^{2} /$ d.o.f. is higher than 2.5 for any $z$ ), by fitting the data with a single absorbed power-law model (typical of obscured AGN) we find a good description of the overall spectrum of the source (for both the observations we obtained a $\chi^{2} /$ d.o.f. $\sim 1$ ). The addition of a thermal component to the power-law model is not statistically required. These results make it unlikely that the X-ray emission is associated with a cluster/group of galaxies, and point towards the presence of a powerful, dominant AGN. Furthermore, as already discussed in Severgnini et al. 2005b and shown in Fig. 3, by fitting the data with an absorbed power-law model, the presence of an excess around $2 \mathrm{keV}$ (observed-frame) suggests line-like residuals. It is worth noting that the line-like residual has been observed only in the second observation where the statistics are better. We have verified that the equivalent width estimated for this line (see Table 2) is comparable to the upper limit measurable in the first observation. The data of the second observation have been fitted again adding a Gaussian component $\left(\Delta \chi^{2}=10\right.$ for $\Delta$ d.o.f. $\left.=3\right)$. Since the strongest feature expected in the X-ray spectrum of AGN is the neutral iron $\mathrm{K} \alpha$ line at $6.4 \mathrm{keV}$ rest-frame (Reynolds et al. 1994), we have associated the $2 \mathrm{keV}$ excess with this line. This places the source at $z=2.27_{-0.35}^{+0.11}$.

The optical VLT spectrum obtained for the object visible in the $R$-band (Fig. 1, left panel) gives a redshift of $z=1.985$ (see Sect. 4). This value is in excellent agreement with the one derived from the XMM-Newton data. We report in Table 2 the best fit (rest-frame) X-ray parameters and the intrinsic X-ray luminosity obtained by fixing the redshift to 1.985 . These values refer to the data of the second observation where the line is observed. Although the quality of the data from the first observation is not good enough to detect the Fe line (we don't use them in this part of the analysis), the continuum parameters obtained from the two observations are consistent within the errors.

On the basis of the optical redshift, the rest-frame energy of the line falls to $5.8_{-0.2}^{+0.7} \mathrm{keV}$. This value is consistent with the 
Table 2. Rest-frame best fit parameters assuming an absorbed power-law at $z=1.985$.

\begin{tabular}{|c|c|c|c|c|c|c|c|c|}
\hline $\begin{array}{l}\text { Obs. ID } \\
011237\end{array}$ & $\Gamma$ & $\begin{array}{c}N_{\mathrm{H}} \\
{\left[10^{22} \mathrm{~cm}^{-2}\right]}\end{array}$ & $\begin{array}{l}E_{\mathrm{Fe}-\mathrm{k} \alpha} \\
{[\mathrm{keV}]}\end{array}$ & $\begin{array}{c}E W_{\mathrm{Fe}-\mathrm{k} \alpha} \\
{[\mathrm{keV}]}\end{array}$ & $\begin{array}{r}\sigma_{\mathrm{Fe}-\mathrm{k} \alpha} \\
{[\mathrm{keV}]}\end{array}$ & $\chi^{2} /$ d.o.f. & $\begin{array}{c}F_{2-10 \mathrm{keV}^{a, c}} \\
{\left[10^{-13} \mathrm{erg} \mathrm{cm}^{-2} \mathrm{~s}^{-1}\right]}\end{array}$ & $\begin{array}{c}L_{2-10 \mathrm{keV}^{b, c}} \\
{\left[10^{44} \mathrm{erg} \mathrm{s}^{-1}\right]}\end{array}$ \\
\hline 2001 & $2.0_{-0.1}^{+0.2}$ & $4.7_{-1.3}^{+1.5}$ & $5.8_{-0.2}^{+0.7}$ & $0.7_{-0.4}^{+0.5}$ & $0.3_{-0.3}^{+0.7}$ & $25.11 / 33$ & 1.1 & 41.0 \\
\hline
\end{tabular}

${ }^{a}$ The flux is corrected only for the Galactic absorption.

${ }^{b}$ The luminosity is corrected for Galactic and intrinsic absorption.

${ }^{c}$ Statistical uncertainties are of about $20 \%$.

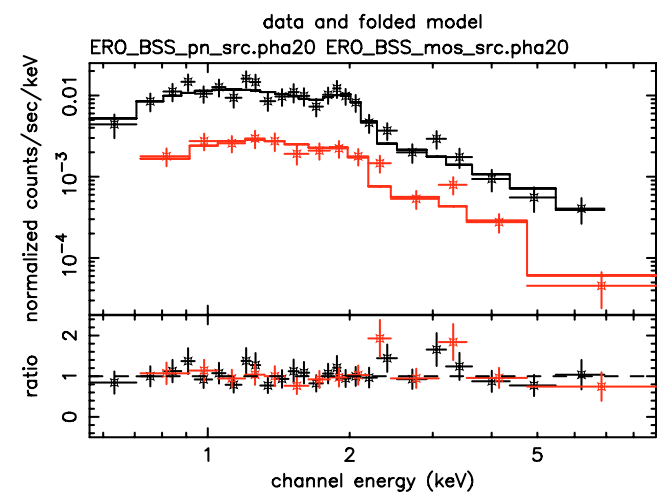

Fig. 4. Mos+pn folded X-ray spectrum of XBS J0216-0435 (upper panel, solid points) and the best-fit for the following model: single absorbed power-law model plus Gaussian line at the optical redshift of $z=1.985$ (continuous lines). Ratio between data and model is plotted in the lower panel as a function of energy.

rest-frame energy of the iron $\mathrm{K} \alpha$ line $(6.4 \mathrm{keV})^{6}$. A detailed analysis of the line properties is not warranted by the quality of the data. Both the line broadening and the high $E W_{\mathrm{Fe}-\mathrm{k} \alpha} \mathrm{ob}-$ served (see Table 2) could be due to the merging of several narrow $(\sigma=0.1 \mathrm{keV}$ ) lines (e.g. $6.4 \mathrm{keV}$ plus $6.7 \mathrm{keV})$. As reported in Table 2, the intrinsic column density measured is typical of obscured AGN $\left(N_{\mathrm{H}} \geq 10^{22} \mathrm{~cm}^{-2}\right)$ and the intrinsic luminosity places the source in the QSO regime $\left(L_{(2-10 \mathrm{keV})}=\right.$ $4.1 \times 10^{45} \mathrm{erg} \mathrm{s}^{-1}$ ).

In conclusion, the $\mathrm{X}$-ray spectral analysis unambiguously reveals the presence of a dominant, high redshift and X-ray obscured QSO.

\section{Optical spectroscopic data}

\subsection{Observation and data reduction}

The object visible in the $R$-band (Fig. 1, left panel) has been observed spectroscopically at ESO VLT using FORS2 during the night between the 29th and the 30th of September 2005 (from UT $=04: 14$ to UT $=06: 14$, including overheads). Grism $300 \mathrm{~V}+20$ was used with no sorting filters and a slit width of $1.6^{\prime \prime}$. The grism gives a spectral dispersion of $3.3 \mathrm{~A} \mathrm{pixel}^{-1}$. The slit was rotated $-20 \mathrm{deg}$ in order to include the second object possibly observed in the $K$-band image. However, only one source turned out to be visible in the spectrum. Two exposures of $2790 \mathrm{~s}$ each have been taken for a total of $5580 \mathrm{~s}$. The DIMM seeing during the observation ranges from $0.90^{\prime \prime}$ to $1.58^{\prime \prime}$ with an average value of about $1.2^{\prime \prime}$. The slit was not oriented along the

\footnotetext{
${ }^{6}$ Two other excesses of lower statistical significance $\left(\Delta \chi^{2}=4\right)$ are present at $\sim 2.37 \mathrm{keV}$ and $3.19 \mathrm{keV}(7.07 \mathrm{keV}$ and $9.52 \mathrm{keV}$ in the restframe assuming a $z=1.985$ ). The first one could be associated to the ionized iron $\mathrm{K} \beta$ line $(7.05 \mathrm{keV})$.
}

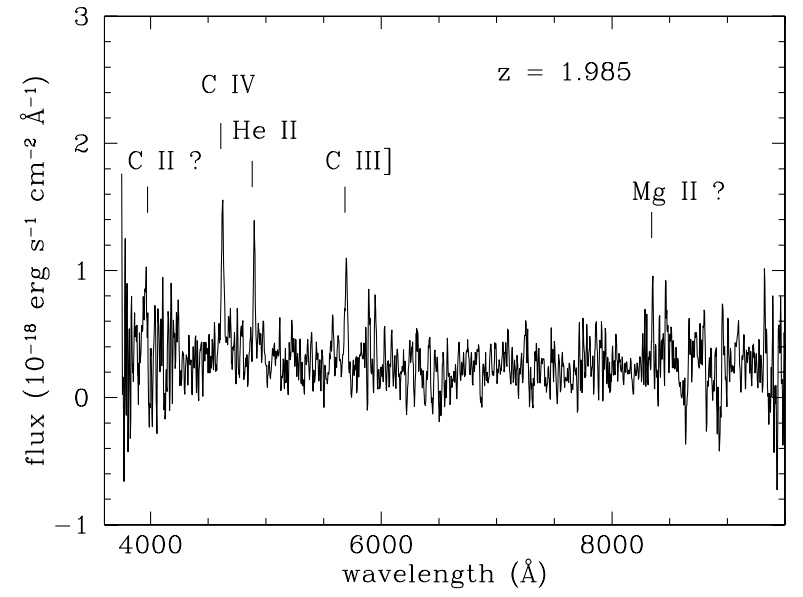

Fig. 5. Low resolution FORS2-VLT optical spectrum of XBS J0216-0435. Data are not corrected for the slit loss.

parallactic angle, but the source was observed at a low airmass (between 1.1 and 1.25) and the slit width was large (1.6") when compared to the average seeing so that only a marginal flux loss in the blue part of the spectrum may have occurred. Therefore, no correction has been applied. For the data reduction we have used the standard IRAF long-slit package following the standard steps.

\subsection{Spectral analysis}

The flux-calibrated spectrum is shown in Fig. 5. At least three emission lines are clearly visible in the spectrum at $4624 \AA$, $4899 \AA$ and $5696 \AA$. These 3 lines are identified with C IV at $\lambda_{\text {rest }}=1549 \AA(z=1.985)$, He II at $1640 \AA(z=1.987)$ and C III] at $1909 \AA(z=1.984)$ respectively. Two weaker additional lines are then possibly identified at $\sim 3960 \AA$ with $\mathrm{C}$ II at $\lambda_{\text {rest }}=$ $1336 \AA$ and at $\sim 8350 \AA$ with $\mathrm{Mg}$ II at $\lambda_{\text {rest }}=2798 \AA$. Using the 3 strongest lines we estimate a redshift of $z=1.985 \pm 0.002$, in excellent agreement with the one derived from X-rays.

The computed widths for the three strongest lines are between $900 \mathrm{~km} \mathrm{~s}^{-1}$ (He II) and 1400-1500 $\mathrm{km} \mathrm{s}^{-1}$ (CIV and $\mathrm{CIII}]$ ). Considering the spectral resolution of the instrument the intrinsic width of the lines is consistent with being $\leq 1200 \mathrm{~km} \mathrm{~s}^{-1}$. This is close to the "standard" classification of type $2 \mathrm{AGN}\left(F W H M<1000 \mathrm{~km} \mathrm{~s}^{-1}\right)$ while it is different from the line widths measured in "Narrow Lined QSO" (NLQSO) like those studied by Baldwin et al. (1988) (1700-2700 $\mathrm{km} \mathrm{s}^{-1}$ ). Another strong indication of the fact that XBS J0216-0435 is a "truly" type 2 QSO and not a QSO where the broad line region produces intrinsically narrow lines, is given by the He II/CIV line ratio. As suggested by Heckman et al. (1995) the He II/CIV line ratio can be used to distinguish between true type 2 objects and narrow line QSO. The former sources show a very 


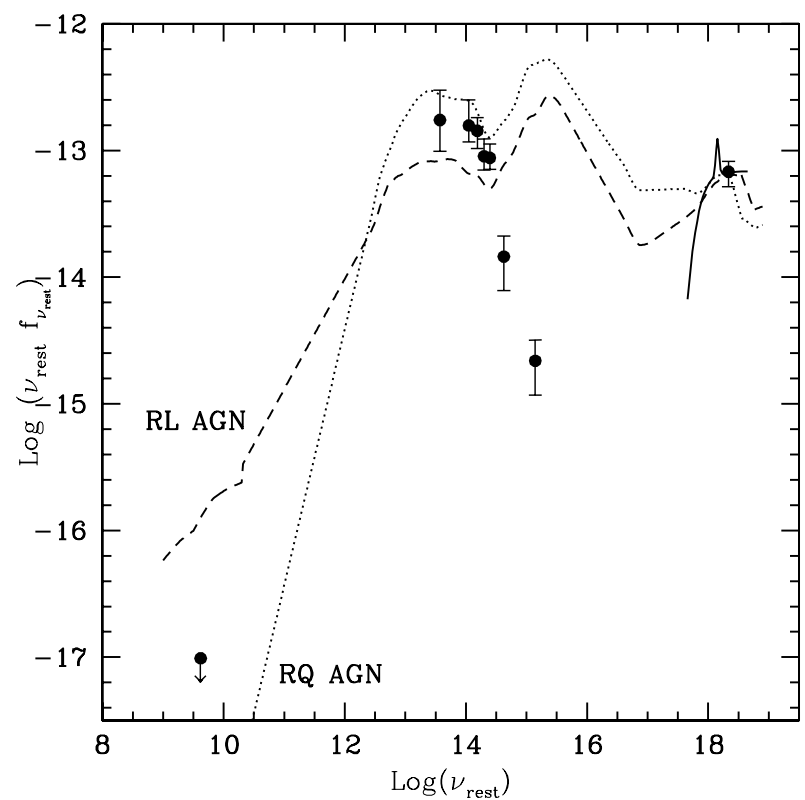

Fig. 6. Spectral energy distribution of XBS J0216-0435. The continuous line is the X-ray best-fit model assuming the PN normalization. Filled symbols are the photometric points in the X-ray, optical, NIR, MIDIR and radio bands (see Sect. 5 for details). The SED of local type 1 radio-loud (dashed line) and radio-quiet (dotted line) QSO (adapted from Elvis et al. 1994) are also shown and normalized to the hard X-ray data of XBS J0216-0435.

strong He II (He II/CIV $\sim 0.6-0.9)$ while in the latters the HeII/CIV ratio is much lower $(\sim 0.05-0.2)$. The optical spectrum of XBS J0216-0435 shows a very prominent He II line (He II/CIV 0.6) in full agreement with the values observed in type 2 AGN (type 2 QSO, Sy2 and Narrow Line radio galaxies).

We conclude that, from the optical spectral point of view, XBS J0216-0435 is a type 2 QSO hosted by an ERO at $z=$ 1.985. In particular, at the time of writing, XBS J0216-0435 is the only type 2 QSO at $z \sim 2$ found in the XMM-BSS survey (the identification rate is $\sim 90 \%$ ). This implies that this kind of sources are quite rare at bright X-ray flux $\left(\sim 10^{-13} \mathrm{erg} \mathrm{s}^{-1} \mathrm{~cm}^{-2}\right)$.

\section{Spectral energy distribution of XBS J0216-0435}

Although the blue spectral coverage of the optical spectrum allows to give only a weak constrain on the absorption in the optical band $\left(A_{\mathrm{V}}\right)$, a more stringent lower limit can be derived by using the spectral energy distribution (SED) of the source. All the available photometric points for XBS J0216-0435 are shown in Fig. 6. While the X-ray, optical and NIR data have been derived from our own analysis (see Sects. 2 and 3.1), the midinfrared (3.6, 4.5, 5.8, 8.0 and $24 \mu \mathrm{m}$ observed-frame) and the radio data $(1.4 \mathrm{GHz}$ observed-frame) are taken from the literature. In particular, the mid-infrared IRAC (the Spitzer Infrared Array Camera; Fazio et al. 2004 - 3.6, 4.5, 5.8, $8 \mu \mathrm{m}$ ) and MIPS (Multi-band Imager for Spitzer; Rieke et al. $2004-24 \mu \mathrm{m}$ ) data are part of the second SWIRE-Spitzer data released catalogue ${ }^{7}$ for the XMM-LSS fields. As for the radio data, the object is covered both by the NVSS (NRAO VLA Sky Survey, Condon et al. 1998) and by the FIRST (Faint Images of the Radio Sky at Twenty-cm, Becker et al. 1995) surveys. In Fig. 6 the $5 \sigma$ upper

\footnotetext{
See http://swire.ipac.caltech.edu/swire/public/ news_archive.html
}

limit on the $1.4 \mathrm{GHz}$ flux density derived from the FIRST survey, that is deeper than the NVSS, is plotted.

We compare the photometry of XBS J0216-0435 with the SED of local type 1 radio-loud (RL) and radio-quiet (RQ) QSOs adapted from Elvis et al. (1994). The two local SEDs have been normalized to the hard X-ray data of XBS J0216-0435. As it is shown from the figure, the X-ray, the MID-IR and the radio photometric points of XBS J0216-0435 are consistent with the SED of RQ-QSO. The optical/NIR data are inconsistent with the SED of type 1 QSO because the optical/NIR photometric points are strongly affected by absorption. By comparing the rest-frame optical flux value measured for XBS J0216-0435 with the corresponding value expected from a RQ unabsorbed AGN we can estimate a more stringent lower limit on the optical absorption. In particular, we find that the intrinsic dust-obscuration associated to our source is $A_{\mathrm{V}}>3 \mathrm{mag}$, typical of type 2 AGN (e.g. Gilli et al. 2001b).

The mid-infrared emission of AGNs is due to the presence of dust tori absorbing and re-radiating a significant fraction of the total luminosity of the primary source. As it is shown from Fig. 6, we find that the near and mid-infrared emission of XBS J0216-0435 is consistent with that of a type 1 QSO. This implies that, if the infrared emission associated to the AGN is not contaminated by other infrared sources (i.e. starlight and/or galactic dust), we don't find evidence of a dependence of the re-radiated infrared continuum with the orientation along the line of sight of the torus (see e.g. Pier \& Krolik 1992; Granato et al. 1997; Efstathiou et al. 1995; Nenkova et al. 2002). In this case, the X-ray/near and mid-infrared flux ratios observed in our source is similar to those of type 1 QSO, extending to high- $z$ similar results obtained for high and low luminosity AGN at lower redshit (Lutz et al. 2004; Sturm et al. 2006).

\section{QSO activity and massive galaxies}

The extended appearance in the optical and NIR bands along with the extremely high X-ray to optical and NIR flux ratios suggest that the host galaxy dominates the emission observed for XBS J0216-0435 at these wavelengths. Taking into account the redshift of the source $(z=1.985)$ and given its apparent magnitude $\left(K^{\prime} \sim 19.5 \mathrm{mag}\right)$, the resulting rest-frame (k-corrected) $K$-band absolute magnitude is $M_{K} \sim-25.5 \mathrm{mag}$, i.e. $\sim 1.6$ times brighter than $L_{K}^{*}$ galaxies at the same $z$ (see Pozzetti et al. 2003; Saracco et al. 2006). Considering $L * \sim 2 \times 10^{11} L_{\odot}$ for galaxies at $z \sim 2$ and assuming a stellar mass-to-light ratio of $0.5(M / L)_{\odot}$, we estimated a stellar mass of the order of $10^{11} M_{\odot}$ for the host galaxy.

This result implies that the powerful QSO2 in XBS J0216-0435 is hosted in a massive system. This source, along with similar results recently obtained for other high- $z$ type 2 QSO (see e.g. Akyiama 2005; Severgnini et al. 2005a; and Maiolino et al. 2006), represents a strong observational evidence of the link between high- $z$ massive galaxies and powerful obscured AGN. The X-ray and optical spectral analysis of a bigger sample of this kind of sources will represent one of the most efficient methods to test a possible co-evolution between massive galaxy and AGN activity (Granato et al. 2004; Di Matteo et al. 2005; Hopkins et al. 2005) and to investigate if and how the AGN feedback can affect the galaxies evolution (see Bower et al. 2005, Stevens et al. 2005). 


\section{Summary and conclusions}

We have presented here the X-ray and optical spectral analysis for one XMM-BSS ERO (XBS J0216-0435) having an extremely high X-ray to optical/NIR flux ratio. This object is the XMM-BSS source with the highest $F_{(2-10 \mathrm{keV})} / F_{\text {opt }}(\sim 220)$. The optical, NIR and X-ray photometric properties of XBS J0216-0435 suggest the presence of an X-ray obscured, optically type 2 QSO.

From the X-ray point of view, the spectral analysis confirms the presence of an obscured AGN. A statistically significant ( $\sim 99 \%)$ excess around $2 \mathrm{keV}$ in the observed-frame suggests the presence of an emission line. By assuming that this feature corresponds to the iron $\mathrm{K} \alpha$ line at $6.4 \mathrm{keV}$ the source is at $z_{X} \sim 2$ with an intrinsic luminosity of the order of $10^{45} \mathrm{erg} \mathrm{s}^{-1}$ and an intrinsic column density of $>10^{22} \mathrm{~cm}^{-2}$, i.e. it is an X-ray obscured QSO.

Dedicated VLT spectroscopic data has allowed us to confirm the spectroscopic redshift $\left(z_{o}=1.985\right)$ derived from the X-ray data. The optical spectral analysis confirms that XBS J02160435 is an absorbed AGN. In conclusion we are dealing with a high redshift, X-ray obscured, optically type 2 QSO.

This source represents one of the few X-ray emitting EROs for which the presence of an X-ray and optical obscured QSO can be unambiguously established (see also Severgnini et al. 2005a; and Maiolino et al. 2006 and references therein). Even more, XBS J0216-0435 can be considered one of the few examples of X-ray obscured QSO2 at high redshift for which a detailed X-ray and optical analysis has been possible and for which a SED from radio to X-rays has been published (see also Sturm et al. 2006).

We want to note that the physical properties derived for XBS J0216-0435 are also in excellent agreement with the relation found by Fiore et al. (2003) for type 2 AGNs. These authors show that there is a correlation between $F_{(2-10 \mathrm{keV})} / F_{\text {opt }}$ and $L_{2-10 \mathrm{keV}}$ for optically obscured AGNs. In particular, they find that higher luminosity obscured AGN have higher $F_{(2-10 \mathrm{keV})} / F_{\text {opt }}$. Following this relation the $F_{\mathrm{X}} / F_{\text {opt }}$ of XBS J0216-0435 is consistent with a $2-10 \mathrm{keV}$ luminosity of the order of $10^{45} \mathrm{erg} \mathrm{s}^{-1}$ in good agreement with what has been found in our analysis.

From the NIR data we have estimated the luminosity and the stellar mass of the host galaxy. An example of the coexistence between powerful QSO and massive galaxy has been found. This result confirms that the selection of X-ray emitting EROs with very high X-ray-to-optical or to-NIR ratio is a very efficient method to find not only high- $z$ QSO but also very massive galaxies and thus to study the fundamental connection between accretion and star-formation processes in the Universe.

Finally, it is worth noting that XBS J0216-0435 is an X-ray line-emitting source like those searched by Maccacaro et al. (2004) and Braito et al. (2005) with the FLEX (Finder of LineEmitting X-ray sources) algorithm in the XLEO (X-ray LineEmitting Object) project. Therefore, a systematic search for XLEO is likely to increase the number of know type 2 QSOs.

Acknowledgements. P. S. acknowledges a research fellowship from the Istituto Nazionale di Astrofisica (INAF). This work has received partial financial support from ASI (ASI/INAF n. I/023/05/0), by the Italian Ministry of Instruction, of University and of Research (MIUR) through grant Cofin-03-02-23. F.J.C. and M.T.C. acknowledge financial support from the Spanish Ministerio de Eduacion y Ciencia under project AYA2003-00812. We thank C. Vignali, F. Cocchia, N. A. Webb for a careful reading of the paper and for useful comments which have improved the paper. The TNG telescope is operated on the island of La Palma by the Centro Galileo Galilei of the INAF in the Spanish Observatorio del Roque de Los Muchachos of the Instituto de Astrofísica de Canarias. We would like to thank the staff members of the ESO and TNG Telescopes for their support during the observations.

\section{References}

Akiyama, M., \& Ohta, K. 2001, PASJ, 53, 63

Akiyama, M. 2005, ApJ, 629, 72

Alexander, D. M., Vignali, C., Bauer, F. E., et al. 2002, AJ, 123, 1149

Alexander, D. M., Bauer, F. E., Brandt, W. N., et al. 2003, AJ, 126, 539

Baldwin, J. A., McMahon, R., Hazard, C., \& Williams, R. E. 1988, ApJ, 327, 103

Barger, A. J., Cowie, L. L., Capak, P., et al. 2003, AJ, 126, 632

Becker, R. H., White, R. L., \& Helfand, D. J. 1995, ApJ, 450, 559

Bower, R. G., Benson, A. J., Malbon, R., et al. 2005, MNRAS, submitted [arXiv: astro-ph/0511338]

Braito, V., Maccacaro, T., Caccianiga, A., Severgnini, P., \& Della Ceca, R. 2005, ApJ, 621, L97

Brusa, M., Comastri, A., Daddi, E., et al. 2005, A\&A, 432, 69

Caccianiga, A., Severgnini, P., Braito, V., et al. 2004, A\&A, 416, 910

Condon, J. J., Cotton, W. D., Greisen, E. W., et al. 1998, AJ, 115, 1693

Della Ceca, R., Maccacaro, T., Caccianiga, A., et al. 2004, A\&A, 428, 383

Della Ceca, R., Caccianiga, A., Severgnini, P., et al. 2005, Proc. of The X-ray Universe 2005, San Lorenzo de El Escorial (Spain) organized by the European Space Astronomy Centre (ESAC) of ESA

Dickey, J. M., \& Lockman, F. J. 1990, ARA\&A, 28, 215

Di Matteo, T., Springel, V., \& Hernquist, L. 2005, Nature, 433, 604

Efstathiou, A., \& Rowan-Robinson, M. 1995, MNRAS, 273, 649,

Elvis, M., Wilkes, B. J., McDowell, J. C., et al. 1994, ApJS, 95, 1

Fazio, G. G., Hora, J. L., Allen, L. E., et al. 2004, ApJS, 154, 10

Fiore, F., Brusa, M., Cocchia, F., et al. 2003, A\&A, 409, 79

Fukugita, M., Shimasaku, K., \& Icikawa, T. 1995, PASP, 107, 945

Gandhi, P., Crawford, C. S., Fabian, A. C., \& Johnstone, R. M. 2004, MNRAS, 348, 529

Gilli, R., Salvati, M., Hasinger, G., et al. 2001a, A\&A, 366, 407

Gilli, R., Risaliti, G., Severgnini, P., et al. 2001b, ASPC, 234, 459

Granato, G. L., Danese, L., \& Franceschini, A. 1997, ApJ, 486, 147

Granato, G. L., De Zotti, G., Silva, L., Bressan, A., \& Danese, L. 2004, ApJ, 600, 580

Heckman, T., Krolik, J., Meurer, G., et al. 1995, ApJ, 452, 549

Hopkins, P. F., Hernquist, L., Cox, T. J., et al. 2005, ApJS, in press [arXiv: astro-ph/0506398]

Kaastra, J. S. 1992, An X-Ray Spectral Code for Optically Thin Plasmas. Internal SRONLeiden Report, updated version 2.0

Liedahl, D. A., Osterheld, A. L., \& Goldstein, W. H. 1995, ApJL, 438, 115

Lutz, D., Maiolino, R., Spoon, H. W. W., \& Moorwood, A. F. M. 2004, A\&A, 418, 465

Maccacaro, T., Gioia, I. M., Wolter, A., Zamorani, G., \& Stocke, J. T. 1988, ApJ, 326, 680

Maccacaro, T., Braito, V., Della Ceca, R., Severgnini, P., \& Caccianiga, A. 2004, ApJ, 617, L33

Maiolino, R., Mignoli, M., Pozzetti, L., et al. 2006, A\&A, 445, 457

Mainieri, V., Bergeron, J., Hasinger, G., et al. 2002, A\&A, 393, 425

Mewe, R., Gronenschild, E. H. B. M., \& van den Oord, G. H. J. 1985, A\&AS, 62, 19

Mignoli, M., Pozzetti, L., Comastri, A., et al. 2004, A\&A, 418, 827

Mohr, J. J., Mathiesen, B., \& Evrard, A. E. 1999, ApJ, 517, 627

Monet, D., Bird, A., Canzian, B., et al. 1998, The PMM USNO A2.0 Catalog (Washington DC: US Naval Observatory)

Nenkova, M., Ivezic, Z., \& Elitzur, M. 2002, ApJ, 570, L9

Pier, E. A., \& Krolik, J. H. 1992, ApJ, 401, 99

Pozzetti, L., Cimatti, A., Zamorani, G., et al. 2003, A\&A, 402, 837

Reynolds, C. S., Fabian, A. C., Makishima, K., Fukazawa, Y., \& Tamura, T. 1994, MNRAS, 268, L55

Rieke, G. H., Young, E. T., Engelbracht, C. W., et al. 2004, ApJS, 154, 25

Roche, N. D., Dunlop, J., \& Almaini, O. 2003, MNRAS, 346, 803

Saracco, P., Fiano, A., Chincarini, G., et al. 2006, MNRAS, 367, 349

Severgnini, P., Caccianiga, A., Braito, V., et al. 2003, A\&A, 406, 483

Severgnini, P., Della Ceca, R., Braito, V., et al. 2005a, A\&A, 431, 87

Severgnini, P., Braito, V., Caccianiga, A., et al. 2005b, Proc. of "The X-ray Universe 2005",

San Lorenzo de El Escorial (Spain) organized by the European Space Astronomy Centre (ESAC) of ESA

Stevens, J. A., Page, M. J., Ivison, R. J., et al. 2005, MNRAS, 360, 610

Sturm, E., Hasinger, G., Lehmann, I., et al. 2006, ApJ, in press

Szokoly, G. P., Bergeron, J., Hasinger, G., et al. 2004, ApJSS, 155, 271

Stevens, J. A., Page, M. J., Ivison, R. J., et al. 2003, MNRAS, 342, 249

Ueda, Y., Akiyama, M., Ohta, K., \& Miyaji, T. 2003, ApJ, 598, 886

Willott, C. J., Simpson, C., Alamini, O., et al. 2003, MNRAS, 339, 397 